\title{
Naloxone protection, social support, network characteristics, and overdose experiences among a cohort of people who use illicit opioids in New York City
}

\author{
Alex S. Bennett ${ }^{1,2^{*}}$ (D) Joy Scheidell ${ }^{2,3}$, Jeanette M. Bowles ${ }^{4}$, Maria Khann ${ }^{2,3}$, Alexis Roth ${ }^{5}$, Lee Hoff ${ }^{3}$,
} Christina Marini ${ }^{1}$ and Luther Elliott ${ }^{1,2}$

\begin{abstract}
Background: Despite increased availability of take-home naloxone, many people who use opioids do so in unprotected contexts, with no other person who might administer naloxone present, increasing the likelihood that an overdose will result in death. Thus, there is a social nature to being "protected" from overdose mortality, which highlights the importance of identifying background factors that promote access to protective social networks among people who use opioids.
\end{abstract}

Methods: We used respondent-driven sampling to recruit adults residing in New York City who reported recent (past 3-day) nonmedical opioid use $(n=575)$. Participants completed a baseline assessment that included past 30-day measures of substance use, overdose experiences, and number of "protected" opioid use events, defined as involving naloxone and the presence of another person who could administer it, as well as measures of network characteristics and social support. We used modified Poisson regression with robust variance to estimate unadjusted and adjusted prevalence ratios (PRs) and 95\% confidence intervals (Cls).

Results: $66 \%$ of participants had ever been trained to administer naloxone, $18 \%$ had used it in the past three months, and $32 \%$ had experienced a recent overdose (past 30 days). During recent opioid use events, $64 \%$ reported never having naloxone and a person to administer present. This was more common among those: aged $\geq 50$ years (PR: 1.18 (Cl 1.03, 1.34); who identified as non-Hispanic Black (PR: 1.27 (Cl 1.05, 1.53); experienced higher levels of stigma consciousness (PR: 1.13 (Cl 1.00, 1.28); and with small social networks (<5 persons) (APR: 1.14 (Cl 0.98, 1.31). Having a recent overdose experience was associated with severe opioid use disorder (PR: 2.45 (Cl 1.49, 4.04), suicidality (PR: 1.72 (Cl 1.19, 2.49), depression (PR: $1.54(\mathrm{Cl} 1.20,1.98)$ and positive urinalysis result for benzodiazepines (PR: 1.56 (Cl 1.23, 1.96), but not with network size.

Conclusions: Results show considerable gaps in naloxone protection among people who use opioids, with more vulnerable and historically disadvantaged subpopulations less likely to be protected. Larger social networks of people who use opioids may be an important resource to curtail overdose mortality, but more effort is needed to harness the protective aspects of social networks.

Keywords: Naloxone protection, Social networks, Non-fatal overdose, Opioids

\footnotetext{
*Correspondence: asb19@nyu.edu

${ }^{1}$ School of Global Public Health, New York University, New York, USA

Full list of author information is available at the end of the article
} permits use, sharing, adaptation, distribution and reproduction in any medium or format, as long as you give appropriate credit to the original author(s) and the source, provide a link to the Creative Commons licence, and indicate if changes were made. The images or other third party material in this article are included in the article's Creative Commons licence, unless indicated otherwise in a credit line to the material. If material is not included in the article's Creative Commons licence and your intended use is not permitted by statutory regulation or exceeds the permitted use, you will need to obtain permission directly from the copyright holder. To view a copy of this licence, visit http://creativecommons.org/licenses/by/4.0/. The Creative Commons Public Domain Dedication waiver (http://creativeco mmons.org/publicdomain/zero/1.0/) applies to the data made available in this article, unless otherwise stated in a credit line to the data. 


\section{Background}

The United States (U.S.) remains in a public health emergency of opioid-related morbidity and mortality, with over 100,000 lives lost in 2021 [1,2]. One of the main public health responses to this epidemic of overdose fatalities has been the widespread implementation of take-home naloxone (THN) programs [3-6]. In New York City (NYC), the setting for this study, naloxone is now widely available at pharmacies, drug treatment programs, syringe service programs (SSPs) [7, 8], mail order, shelters, prisons, jails, and through many communitybased programs [9]. THN programs equip people who use drugs and interested community members with both naloxone and the knowledge to prevent (e.g. through avoiding concurrent use of opioids and alcohol or benzodiazepines) and respond to overdose events. Despite widespread access to THN for people who use drugs in places like NYC, there remain important open questions about the contexts in which THN availability does not equate to greater protection from overdose death.

THN overdose protection is contingent on consuming opioids in the presence of another person equipped with and capable of administering intranasal or intramuscular naloxone, as it cannot presently be reliably self-administered. As such, consuming drugs alone significantly heightens risk for overdose death [10]. Research examining the settings of overdose found that up to $75 \%$ of overdose fatalities in NYC occurred in private residences [11], placing the burden of responsibility for response on others in the household, if any exist. Ideally, others in the household would be trained in THN programs, observe or be aware of drug consumption events, and possess naloxone. Most people who witness overdoses are other people who use drugs [5]. However, even among people who use drugs and are trained in THN programs, they do not always carry naloxone. To illustrate, a study from Baltimore found that among longtime heroin users, twothirds reported ever receiving naloxone, but of those only one-quarter reported always carrying it [12]. Existing research has identified many reasons why people who use drugs might not carry naloxone, thus limiting overdose protectedness, which include: stigma [13], ambivalence [13], insecurity about competence to use it [14], fear of police involvement, concerns about unnecessarily precipitating withdrawal [15], or consuming drugs alone. For people who primarily consume drugs alone, outside of networks of other people who use drugs and share the responsibility of overdose response, the value of THN is effectively negated.

There is a range of reasons why people might consume drugs alone, including the desire to avoid the stigmatizing judgment of others, safety, comfort, distrust of others, being pressured to share drugs, previous harmful experiences particularly when over-sedated, and drug use preferences [13, 16-18]. Using alone denies individuals the benefits identified with membership in social networks, which include: facilitating collective drug purchasing, group safety, resource sharing, maintaining group surreptitiousness, and social, economic and emotional support $[17,19]$. Research has demonstrated how networks of people who use drugs can be resources for one another helping prevent many drug-related harms, thus creating a "social capital" that facilitates overdose response [20, 21]. Accordingly, the size of one's social network of people who use drugs might be a critical indicator of their protectedness against an overdose fatality. To that end, the present study aims to uncover various factors associated with a person's opioid-using network size and its relationship to their number of opioid use events during which another person and naloxone were present, thus representing a form of "protected use" from fatal overdose.

\section{Methods \\ Study design and sample}

To begin to explore the relationship between different types of social network supports and naloxone availability during overdose events, we developed a measure of overdose protectedness, reflecting the proportion of opioid use events in the past 30 days in which both a trusted person and naloxone were available. In the current analysis, we present descriptive statistics related to THN training and possession histories and examined potential associations between network characteristics, overdose protectedness, and non-fatal opioid-involved overdose among 575 people $(n=575)$ who use illicit opioids in NYC.

Participants enrolled in the study were persons aged 18 or older who were currently (defined as within the past 3 days) using an unprescribed opioid (e.g. heroin, fentanyl, and prescription opioids). Self-report of opioid use was verified using a rapid urinalysis tool from BTNX that included 9 fentanyl-class drugs in addition to heroin/ morphine, benzodiazepines, alcohol, amphetamines, oxycodone, marijuana, and methadone metabolites. Recruitment followed a traditional respondent-driven sampling (RDS) approach [22, 23] that used numbered coupons to allow participants to refer up to three of their opioid-using network members to the study. Ten "seeds" representing ethnic, gender, and geographical diversity were established, ultimately resulting in a sample of 575 participants.

Once determined to be eligible and having provided informed consent, participants completed a face-toface baseline survey instrument administered by a trained and experienced interviewer entering data on 
a tablet computer. Upon completion of baseline assessment, participants were trained in THN, which included a12-minute NYC Department of Health training video. Participants were compensated $\$ 60$ for their time (approximately $2.5 \mathrm{~h}$ ) and given 3 numbered coupons that they were asked to distribute to other people who use opioids. Participants were given an additional \$15 for each referral of an eligible participant that resulted in a completed enrollment. Due to the COVID-19 pandemic, recruitment stopped in early March of 2020, roughly one week in advance of completing the target sample of $N=600$. All procedures were approved by the Institutional Review Board of the host institution.

\section{Measures}

\section{Network size}

Opioid-using network members were defined using the inclusion criteria for the study. We measured baseline network size using two indicators: 1) the participantreported number of adults ( $>18$ years of age) whose names they knew (and who also knew their name) that live in NYC and were known to have used illicit opioids in the last three days, and 2) whom the participant had seen at least once in the past two weeks. Because the recruitment of participants was network-based, all participants had at least one network member (the person who had referred them to the study by providing an RDS coupon), and we considered those with less than five reported members in their opioid-using networks to have small networks. Among those who inject drugs $(n=102)$, we also measured the total number of people who had been in their presence when injecting drugs in the past three months, which we dichotomized as $\geq 1$ versus 0 to capture isolated injection.

\section{Social support}

Because the size of social networks is an imprecise indicator of how intimate or supportive these networks are, we included items about the frequency of reported forms of social and material support they had received from their non-drug-using (e.g., "your relatives, nondrug using friends and/or neighbors, program staff") and drug-using network members in the past three months, including offers of a place to sleep, gifts of money with no strings attached, and emotional support when they were unhappy. We created separate dichotomous past 30-day indicators for each. To investigate how the constitution of networks may relate to overdose risk and protection, we also created a combined categorical support indicator defined as having received none of the forms of support from using and non-using networks; receiving $\geq 1$ form of support from drug-using but no support from non-using network; receiving $\geq 1$ form of support from non-using but no support from drug-using network; and receiving $\geq 1$ form of support from both networks. Finally, participants reported if they were currently cohabiting with a romantic partner, cohabiting with anyone, and if that cohabiting person used opioids.

\section{Protection with naloxone}

In the survey, participants reported the number of days they had used opioids in the past 30 days and the number of times they used, on average, each day, which we multiplied to estimate the total number of past 30-day opioid use events. Participants also reported the number of opioid use events in the past 30 days during which both naloxone and a person trusted to administer it were present, which are defined as "protected use" events. To calculate the proportion of protected use events in the past 30 days, we divided the reported number of opioid use events where naloxone and a person were present by the total reported number of opioid use events. We multiplied this proportion by 100 to capture the percentage of protected events, which we then dichotomized as never protected (e.g., $0 \%$ protected events) versus at least some protected events (e.g., $>0 \%$ protected events) in the past 30 days.

\section{Opioid overdose}

Past-month indicators of opioid-related overdose were drawn from the Recent Overdose Experience Scale (RODES) [24]. Empirically-derived estimates suggest that less than $5 \%$ of heroin-related overdoses are fatal [25, 26] making non-fatal overdoses a critical outcome for research, particularly where sample size is constrained. The RODES assesses a number of clinical indicators of opioid-involved non-fatal overdose, including loss of consciousness, labored breathing, face or fingers turning blue, emergency services called, or naloxone administered, as well as asking respondents to estimate the number of days in the past month during which they believed they had an opioid-involved overdose, defined broadly as an event during which "you were more sedated drugged, or high than you wanted to be or felt was safe." We considered an affirmative response to any of these experiences to indicate an overdose experience.

\section{Sample characteristics and covariates}

Queried correlates included self-reported sociodemographic characteristics of age; race/ethnicity (categorized as non-Hispanic white, non-Hispanic Black, Hispanic/ Latinx, and other e.g., Asian, American Indian or Native American, Native Hawaiian/Pacific Islander); sex (categorized as male/trans male and female/trans female); and employment status (categorized as employed full- or part-time, off books in informal/cash jobs, homemaker, 
or full-time student versus unemployed or unable to work for health reasons). Participants reported if they were currently homeless and if they had ever been incarcerated. Opioid use disorder (OUD) severity was measured using the Diagnostic and Statistical Manual of Mental Disorders Version 5 (DSM 5) criteria [27] and categorized as mild (score 2-3), moderate (score 4-5), or severe (score $\geq 6$ ). Participants reported if they had ever injected heroin, as well as past 30-day use of cocaine, crack, marijuana, and/or benzodiazepine. Drug userelated stigma was measured using six items adapted from the Stigma Consciousness Scale [28], on which participants reported, using a Likert-type response scale, their level of agreement with statements such as, "I worry that other people might find out that I use drugs" and "I feel guilty about using drugs." We summed responses to the six items (range $0-24$, alpha $=0.76$ ) and dichotomized at the sample median (scores $\geq 13$ ). Posttraumatic stress disorder (PTSD) symptoms were measured using the six-item version of the PTSD Checklist [29] (range $0-24$, alpha $=0.90$ ), with scores $\geq 14$ considered a positive screen for PTSD. Overall mental health was measured as a continuous score capturing the participants' average across the 20 items in the DSM-5 Cross-Cutting Symptom scale (range 0-4; Cronbach's alpha $=0.91$ ); depression, anxiety, and suicidality were defined based on subscales [30]. The frequency with which participants visited a syringe exchange program (SEP) in the past three months was coded as "never," "less than once a month," and "almost daily/daily." Participants reported if they were currently receiving treatment for opioid use and, if so, what form of treatment. Those receiving treatment were all receiving medication-assisted treatment, hence we created a dichotomous measure of no current treatment versus medication-assisted treatment. Finally, participants reported if they had ever been trained to administer naloxone and if they had administered it to anyone in the past three months.

\section{Analyses}

All analyses were conducted in Stata 15.1 [31]. In univariate analyses, we estimated the frequency and proportion of sample characteristics in the total sample. In bivariate analyses, we estimated the frequency and proportion of having fewer than five members in one's opioid network, of having no social support from using network members, of having $0 \%$ protected opioid use events, and of overdose experiences for each sample characteristic. We used a modified Poisson regression with robust variance estimation to estimate prevalence ratios (PRs) and 95\% confidence intervals (CIs) for association. We used modified Poisson regression because our binary outcomes were not rare [32] and because this method provides statistically principled estimates when conducting regression analyses with data from an RDS study design [33]. For each indicator of network size, social support and cohabiting, we estimated the frequency and proportion of past 30 days: (1) events of using opioids without naloxone and a person present, and (2) having had an opioid overdose. We estimated unadjusted and adjusted PRs, with adjusted models controlling for age, sex, race/ ethnicity, current homelessness, and OUD severity.

\section{Results}

Participant socio-demographic characteristics, drug use, and drug treatment and harm reduction access (Table 1) Of the study population of opioid users, $65.8 \%$ were men and $33.5 \%$ were women; their mean age was 48.3 years with $55.7 \% 50$ years of age or older; and $39.8 \%$ were Hispanic/Latinx, 37.7\% were non-Hispanic Black, 18.4\% were non-Hispanic white, and 3.3\% were categorized as another racial/ethnic background (Tables 1,2). The majority of the sample were unemployed (77.3\%)and had a history of incarceration (80.2\%). Thirty percent of participants reported they were currently homeless. The majority of those not experiencing homelessness lived with someone else (75.6\%), with one-quarter reporting cohabitation with a romantic partner (25.9\%) and $17.7 \%$ reported cohabitating with a romantic partner used opioids. Over one-quarter cohabited some someone who uses opioids, including romantic partners (28.0\%).

Based on DSM- 5 criteria, the majority of the sample had severe OUD $(76.6 \%)$, with $34.0 \%$ reporting lifetime history of heroin injection. Urine-based toxicology indicated $85.9 \%$ had recent exposure to heroin, $53.1 \%$ to fentanyl, and $5.7 \%$ to oxycodone. Substantial proportions reported use of other drugs in the past 30 days including cocaine $(50.0 \%)$, crack $(28.0 \%)$, marijuana $(37.8 \%)$, and a benzodiazepine (32.8\%). Participants reported experiencing drug-related stigma (48.6\%), depressive symptoms (54.1\%), anxiety symptoms (45.4\%), PTSD (10.8\%), and suicidal ideation (4.9\%). Only $29.0 \%$ reported SEP use. Of the 575 recruited participants, $65.8 \%$ had ever been trained to use naloxone, and $17.7 \%$ had administered it in the past three months.

Of those trained to use naloxone $(n=379), 80 \%$ $(302 / 379)$ were in possession of a THN kit at the time of the interview. On average, participants reported using 4.4 times (standard deviation [SD] 7.6) per day in the past 30 days and using on 23.6 days (SD 9.0) of the past 30 days; the mean number of use events in the days 30 days was 104.0 (SD 163.8). The mean number of days naloxone was reported to be available when using opioids in the past 30 days was 7.9 days. When asked how often naloxone was available during opioid use events in the past 30 days, $45 \%$ of all participants reported naloxone 
Table 1 Participant characteristics in the total sample and by network characteristics $(N=575)$

\begin{tabular}{|c|c|c|c|c|c|}
\hline & $N(\%)$ in total sample ${ }^{a}$ & $\begin{array}{l}N(\%) \text { with }<5 \\
\text { members in } \\
\text { network }^{\text {b }}\end{array}$ & $\begin{array}{l}\text { PR }(95 \% \mathrm{Cl}) \text { for } \\
\text { association with }<5 \\
\text { members in network }\end{array}$ & $\begin{array}{l}N(\%) \text { with no support } \\
\text { from using network } \\
\text { members }\end{array}$ & $\begin{array}{l}\text { PR }(95 \% \mathrm{Cl}) \text { for } \\
\text { association with no } \\
\text { support from using } \\
\text { network }^{\mathrm{e}}\end{array}$ \\
\hline \multicolumn{6}{|l|}{ Age } \\
\hline Less than 50 years of age & $255(44.3)$ & $68(26.8)$ & Ref & $103(40.6)$ & Ref \\
\hline 50 years of age or older & $321(55.7)$ & $63(19.6)$ & $0.73(0.54,0.99)$ & $156(48.8)$ & $1.20(1.00,1.45)$ \\
\hline \multicolumn{6}{|l|}{ Race/ethnicity } \\
\hline Non-Hispanic White & $106(18.4)$ & $26(24.8)$ & Ref & $46(43.4)$ & Ref \\
\hline Non-Hispanic Black & $217(37.7)$ & $47(21.7)$ & $0.87(0.58,1.33)$ & $108(49.8)$ & $1.15(0.89,1.48)$ \\
\hline Hispanic/Latinx & $229(39.8)$ & $53(23.1)$ & $0.93(0.62,1.41)$ & $97(42.7)$ & $0.98(0.76,1.28)$ \\
\hline Other & $19(3.30)$ & $4(21.0)$ & $0.85(0.33,2.16)$ & $6(31.6)$ & $0.73(0.36,1.46)$ \\
\hline \multicolumn{6}{|l|}{ Sex } \\
\hline Female & $193(33.5)$ & $43(22.5)$ & Ref & $90(27.1)$ & Ref \\
\hline Male & $379(65.8)$ & $87(23.0)$ & $1.02(0.74,1.41)$ & $167(44.3)$ & $0.94(0.78,1.13)$ \\
\hline \multicolumn{6}{|l|}{ Employment status } \\
\hline Unemployed & $445(77.3)$ & 99 (22.3) & Ref & $195(44.0)$ & Ref \\
\hline Employed & $124(21.5)$ & $30(24.2)$ & $1.08(0.76,1.55)$ & $62(50.0)$ & $1.14(0.92,1.39)$ \\
\hline \multicolumn{6}{|l|}{ Currently homeless } \\
\hline No & $402(69.9)$ & $90(22.4)$ & Ref & $184(45.9)$ & Ref \\
\hline Yes & $173(30.1)$ & $41(23.7)$ & $1.06(0.76,1.46)$ & $75(43.4)$ & $0.76(0.63,0.93)$ \\
\hline \multicolumn{6}{|l|}{ Everincarcerated } \\
\hline No & $114(19.8)$ & $33(29.0)$ & Ref & $63(55.8)$ & Ref \\
\hline Yes & $462(80.2)$ & $98(21.3)$ & $0.73(0.52,1.03)$ & $196(42.5)$ & $0.59(0.39,0.89)$ \\
\hline \multicolumn{6}{|c|}{ Currently lives with romantic partner } \\
\hline No & $426(74.1)$ & $103(24.2)$ & Ref & $192(45.3)$ & Ref \\
\hline Yes & $149(25.9)$ & $28(18.8)$ & $0.78(0.53,1.13)$ & $67(44.7)$ & $0.99(0.80,1.21)$ \\
\hline \multicolumn{6}{|c|}{ Currently lives with romantic partner who uses opioids } \\
\hline No & $473(82.3)$ & $115(24.3)$ & Ref & $215(45.6)$ & Ref \\
\hline Yes & $102(17.7)$ & $16(15.7)$ & $0.64(0.40,1.04)$ & $44(42.7)$ & $0.94(0.73,1.20)$ \\
\hline \multicolumn{6}{|c|}{ Currently lives with anyone } \\
\hline No & $140(24.4)$ & $30(21.4)$ & Ref & $67(48.2)$ & Ref \\
\hline Yes & $434(75.6)$ & $101(23.3$ & $1.09(0.76,1.56)$ & $192(44.2)$ & $0.92(0.75,1.12)$ \\
\hline \multicolumn{6}{|c|}{ Currently lives with anyone who uses opioids } \\
\hline No & $399(72.0)$ & $98(24.6)$ & Ref & $186(46.8)$ & Ref \\
\hline Yes & $155(28.0)$ & $27(17.4)$ & $0.71(0.48,1.04)$ & $64(41.0)$ & $0.88(0.70,1.09)$ \\
\hline \multicolumn{6}{|l|}{ Current living situation } \\
\hline Lives alone & $140(25.3)$ & $30(21.4)$ & Ref & $67(48.2)$ & Ref \\
\hline $\begin{array}{l}\text { Not with anyone who } \\
\text { uses opioids }\end{array}$ & $259(46.8)$ & $68(26.2)$ & $1.22(0.84,1.79)$ & $119(46.1)$ & $0.96(0.77,1.19)$ \\
\hline $\begin{array}{l}\text { Someone who uses } \\
\text { opioids }\end{array}$ & $155(28.0)$ & $27(17.4)$ & $0.81(0.51,1.30)$ & $64(41.0)$ & $0.85(0.66,1.10)$ \\
\hline \multicolumn{6}{|l|}{ Length of opioid use history } \\
\hline 15 years of less & $154(16.8)$ & $46(30.1)$ & Ref & $71(46.4)$ & Ref \\
\hline $16-26$ years & $136(23.6)$ & $30(22.1)$ & $0.73(0.49,1.09)$ & $52(38.2)$ & $0.82(0.63,1.08)$ \\
\hline $27-35$ years $27-35$ years & $147(25.6)$ & $32(21.8)$ & $0.72(0.49,1.07)$ & $72(49.0)$ & $1.06(0.83,1.34)$ \\
\hline 36 years or longer & $137(23.8)$ & $23(16.8)$ & $0.56(0.36,0.87)$ & $64(47.1)$ & $1.01(0.79,1.30)$ \\
\hline \multicolumn{6}{|l|}{ OUD severity } \\
\hline Mild/moderate & $95(16.5)$ & $24(25.3)$ & Ref & $56(59.0)$ & Ref \\
\hline Severe & $441(76.6)$ & $93(21.1)$ & $0.84(0.57,1.24)$ & $186(42.4)$ & $0.72(0.59,0.88)$ \\
\hline \multicolumn{6}{|l|}{ Ever injected heroin } \\
\hline No & $365(63.4)$ & $85(23.3)$ & Ref & $175(48.2)$ & Ref \\
\hline
\end{tabular}


Table 1 (continued)

\begin{tabular}{|c|c|c|c|c|c|}
\hline & $N(\%)$ in total sample ${ }^{a}$ & $\begin{array}{l}N(\%) \text { with }<5 \\
\text { members in } \\
\text { network }^{\text {b }}\end{array}$ & $\begin{array}{l}\text { PR }(95 \% \mathrm{Cl}) \text { for } \\
\text { association with }<5 \\
\text { members in network }\end{array}$ & $\begin{array}{l}N(\%) \text { with no support } \\
\text { from using network } \\
\text { members }\end{array}$ & $\begin{array}{l}\text { PR }(95 \% \mathrm{Cl}) \text { for } \\
\text { association with no } \\
\text { support from using } \\
\text { network }^{\mathrm{e}}\end{array}$ \\
\hline Yes & $196(34.0)$ & $39(20.0)$ & $0.86(0.61,1.20)$ & $75(38.3)$ & $0.80(0.64,0.98)$ \\
\hline \multicolumn{6}{|c|}{ Cocaine use in past 30 days } \\
\hline No & $288(50.0)$ & $77(26.7)$ & Ref & $139(48.4)$ & Ref \\
\hline Yes & $288(50.0)$ & $54(18.8)$ & $0.70(0.52,0.96)$ & $120(41.8)$ & $0.86(0.72,1.04)$ \\
\hline \multicolumn{6}{|l|}{ Crack use in past 30 days } \\
\hline No & $415(72.0)$ & $95(23.0)$ & Ref & $197(47.6)$ & Ref \\
\hline Yes & $161(28.0)$ & $26(22.4)$ & $0.97(0.70,1.37)$ & $62(38.8)$ & $0.81(0.65,1.01)$ \\
\hline \multicolumn{6}{|c|}{ Marijuana use in past 30 days } \\
\hline No & $358(62.2)$ & $84(23.5)$ & Ref & $170(47.6)$ & Ref \\
\hline Yes & $218(37.8)$ & $47(21.6)$ & $0.92(0.67,1.26)$ & $89(41.1)$ & $0.86(0.71,1.04)$ \\
\hline \multicolumn{6}{|c|}{ Benzodiazepine use in past 30 days } \\
\hline No & $387(67.2)$ & $88(22.8)$ & Ref & $178(46.2)$ & Ref \\
\hline Yes & $189(32.8)$ & $43(22.8)$ & $1.00(0.72,1.38)$ & $81(42.9)$ & $0.93(0.76,1.13)$ \\
\hline \multicolumn{6}{|c|}{ Fentanyl detected in urine analysis } \\
\hline No & $268(46.5)$ & $64(23.9)$ & Ref & $135(50.6)$ & Ref \\
\hline Yes & $306(53.1)$ & $66(21.6)$ & $0.90(0.67,1.23)$ & $123(40.3)$ & $0.80(0.66,0.96)$ \\
\hline \multicolumn{6}{|c|}{ Oxycodone detected in urine analysis } \\
\hline No & $541(93.9)$ & $123(22.8)$ & Ref & $247(45.7)$ & Ref \\
\hline Yes & $33(5.7)$ & $7(21.2)$ & $0.93(0.47,1.83)$ & $11(34.4)$ & $0.75(0.46,1.22)$ \\
\hline \multicolumn{6}{|c|}{ Heroin detected in urine analysis } \\
\hline No & $79(13.7)$ & $17(21.5)$ & Ref & $43(55.1)$ & Ref \\
\hline Yes & $495(85.9)$ & $113(22.9)$ & $1.06(0.68,1.67)$ & $215(43.5)$ & $0.79(0.63,0.99)$ \\
\hline \multicolumn{6}{|l|}{ Stigma (median $\geq 13$ ) } \\
\hline No & $278(48.3)$ & $53(19.1)$ & Ref & $120(43.2)$ & Ref \\
\hline Yes & $280(48.6)$ & $68(24.4)$ & $1.27(0.93,1.76)$ & $127(45.5)$ & $1.05(0.88,1.27)$ \\
\hline \multicolumn{6}{|l|}{ PTSD } \\
\hline No & $513(89.1)$ & $117(22.8)$ & Ref & $232(45.4)$ & Ref \\
\hline Yes & $62(10.8)$ & $14(22.6)$ & $0.99(0.61,1.61)$ & $27(43.6)$ & $0.96(0.71,1.29)$ \\
\hline \multirow[t]{2}{*}{ Omnibus mental health* } & $0.7(0.25,1.3)$ & & Ref & & Ref \\
\hline & & & $1.02(0.84,1.24)$ & & $0.99(0.87,1.12)$ \\
\hline \multicolumn{6}{|l|}{ Depression } \\
\hline No & $264(45.9)$ & $62(23.5)$ & Ref & $115(43.6)$ & Ref \\
\hline Yes & $69(54.1)$ & $69(22.2)$ & $0.94(0.70,1.28)$ & $114(46.4)$ & $1.07(0.89,1.28)$ \\
\hline \multicolumn{6}{|l|}{ Anxiety } \\
\hline No & $314(54.6)$ & $70(22.3)$ & Ref & $143(45.5)$ & Ref \\
\hline Yes & $216(45.4)$ & $61(23.4)$ & $1.05(0.78,1.42)$ & $116(44.6)$ & $0.98(0.82,1.17)$ \\
\hline \multicolumn{6}{|l|}{ Suicidality } \\
\hline No & $546(95.1)$ & $125(22.9)$ & Ref & $247(45.2)$ & Ref \\
\hline Yes & $28(4.9)$ & $6(21.4)$ & $0.94(0.45,1.94)$ & $12(44.4)$ & $0.98(0.64,1.51)$ \\
\hline \multicolumn{6}{|c|}{ SEP visits in past 3 months } \\
\hline No & $347(60.2)$ & $88(25.4)$ & Ref & $172(49.7)$ & Ref \\
\hline Yes & $170(29.5)$ & $30(17.8)$ & $0.70(0.48,1.02)$ & $64(17.9)$ & $0.76(0.51,0.95)$ \\
\hline \multicolumn{6}{|c|}{ Currently opioid treatment } \\
\hline No current treatment & $254(44.2)$ & $71(28.0)$ & Ref & $104(41.1)$ & Ref \\
\hline $\begin{array}{l}\text { Medication assisted } \\
\text { treatment }\end{array}$ & $321(55.8)$ & $60(18.7)$ & $0.67(0.49,0.90)$ & $155(48.3)$ & $1.17(0.98,1.41)$ \\
\hline
\end{tabular}


Table 1 (continued)

\begin{tabular}{|c|c|c|c|c|c|}
\hline & $N(\%)$ in total sample ${ }^{a}$ & $\begin{array}{l}N(\%) \text { with }<5 \\
\text { members in } \\
\text { network }^{b}\end{array}$ & $\begin{array}{l}\text { PR }(95 \% \mathrm{Cl}) \text { for } \\
\text { association with }<5 \\
\text { members in network }\end{array}$ & $\begin{array}{l}N(\%) \text { with no support } \\
\text { from using network } \\
\text { members }\end{array}$ & $\begin{array}{l}\text { PR }(95 \% \mathrm{Cl}) \text { for } \\
\text { association with no } \\
\text { support from using } \\
\text { network }^{\mathrm{e}}\end{array}$ \\
\hline \multicolumn{6}{|c|}{ Ever trained to administer naloxone } \\
\hline No & $193(33.5)$ & $56(29.0)$ & Ref & $95(49.5)$ & Ref \\
\hline Yes & $379(65.8)$ & $93(19.3)$ & $0.66(0.49,0.90)$ & $163(43.1)$ & $0.87(0.72,1.05)$ \\
\hline \multicolumn{6}{|c|}{ Administered naloxone in past 3 months } \\
\hline No & $474(82.3)$ & $120(25.4)$ & Ref & $227(48.1)$ & Ref \\
\hline Yes & $102(17.7)$ & $11(10.8)$ & $0.42(0.24,0.76)$ & $32(31.4)$ & $0.65(0.48,0.88)$ \\
\hline
\end{tabular}

${ }^{\text {a }}$ May not sum to 575 due to missing values

${ }^{\mathrm{b}} \mathrm{N}=575 ; 5$ members is the 25th percentile [131 (22.7\%) with $<5$ members; 444 (77.1\%) with $5+$ members]

c Compared to $5+$ Members

${ }^{\mathrm{d}} N=575$ [259 (45.0\%) have no material or emotional support; $315(54.7 \%)$ have at least one form of support]

e Compared to those with at least one form of support

" Median (IQR); scale is standardized

was never available during opioid use, $35 \%$ it was sometimes, and $20 \%$ reported it was always available.

\section{Network size and support by respondent characteristics (Table 1)}

The prevalence of respondents with a small network (in the bottom $25^{\text {th }}$ percentile for number in the network; $<5$ members) was $22.7 \%$ (Table 1). There was evidence that the prevalence of having a small network was lower in those who were 50 years or older (PR: 0.73 ; 95\% CI 0.54, 0.99), who lived with either a romantic partner or any person who uses opioids (partner: PR: 0.64, 95\% CI 0.40, 1.04; any person: $0.71,95 \%$ CI $0.48,1.04$ ). Small network size also was less likely for those in MAT (PR: 0.67, 95\% CI $0.49,0.90)$ and among those who were trained in and had administered naloxone (trained: PR: $0.66,95 \% \mathrm{CI}$ 0.49, 0.90); (administered: PR: 0.42, 95\% CI 0.24, 0.76). Network size was not correlated with gender, race/ethnicity, socio-economic factors, drug use patterns, or mental health factors.

The prevalence of respondents with no material or emotional support (from either drug using network members or non-drug using network members) was $45.0 \%$ (Table 1). A lack of support was more common among those aged 50 years and older (PR: 1.20 ; 95\% CI $1.00,1.45$ ) (Table 1). Race/ethnicity, gender, and employment were not correlated with having or not having support. Lack of support was less common among respondents who were homeless (PR: 0.76; 95\% CI 0.63, $0.93)$, those with a history of incarceration $(P R=0.59$; $95 \%$ CI $0.39,1.45$ ), and those with severe OUD (PR: 0.72 , $0.59,0.88)$. Lack of support also was less likely among those who attended SEPs (PR: 0.76, 95\% CI 0.51, 0.95) and who were trained in and had administered naloxone (trained: PR: 0.87, 95\% CI 0.72, 1.05); (administered: PR: $0.65,95 \%$ CI $0.48,0.88)$.

\section{Overdose risk by respondent characteristics (Table 2)}

When asked how often both naloxone and a person trusted to administer it were present during opioid use events in the past 30 days, $65 \%$ reported both naloxone and a person to administer it was never available during opioid use and hence were considered $0 \%$ protected from fatal overdose during those events. Having $0 \%$ overdose protection was more common among those aged 50 years and older (PR: 1.18, 95\% CI 1.03, 1.34), non-Hispanic Black respondents (PR: 1.27, CI 1.05, 1.53) was less likely among opioid users who had severe OUD (PR: 0.86, 95\% CI $0.74,1.00$ ), who injected heroin (PR: $0.71,95 \%$ CI 0.61 , 0.83 ), and who tested positive for fentanyl (95\% CI 0.86 , $95 \%$ CI $0.76,0.98)$. There was also evidence that $0 \%$ protection was less common among those who used other substances including cocaine (PR: $0.86,95 \%$ CI $0.76,0.98$ ) and benzodiazepines (PR: $0.89,95 \%$ CI $0.78,1.02$ ) and who had anxiety symptoms (PR: $0.89,95 \%$ CI $0.78,1.01$ ). Those who were trained in and who had administered naloxone also were less likely to be $0 \%$ protected during opioid use (trained: PR: 0.62, 95\% CI 0.56, 0.70); (administered: PR: $0.58,95 \%$ CI $0.45,0.74$ ).

A total of 185 (32\%) had an OD experience in the past 30 days at baseline. Having experienced an overdose was less common among those aged 50 years and older (PR: 0.60, 95\% CI 0.47, 0.77) and non-Hispanic Black respondents (PR: 0.62, 95\% CI 0.46, 0.85) and was more common among those who were employed (PR: $1.44,95 \%$ CI 1.12, 1.84). Overdose experience was more common among opioid users who had severe OUD (PR: 2.45; 95\% CI 1.49, 4.04), and those who injected heroin 
Table 2 Participant characteristics in the total sample and by unprotected use and OD experience in past 30 days $(N=575)$

$\begin{array}{llll}\begin{array}{l}N(\%) \text { with } \\ \text { unprotected use }\end{array} & \begin{array}{l}\text { PR }(95 \% \mathrm{Cl}) \text { for association } \\ \text { unprotected use }\end{array} & \begin{array}{l}N(\%) \text { with OD } \\ \text { experience }\end{array} & \begin{array}{l}\text { PR }(95 \% \mathrm{Cl}) \text { for } \\ \text { association with OD } \\ \text { experience }\end{array}\end{array}$

\section{Age}

Less than 50 years of age

50 Years of age or older

Race/ethnicity

Non-Hispanic White

Non-Hispanic Black

Hispanic/Latinx

Other

Sex

Female

Male

Employment status

Unemployed

Employed

Currently homeless

No

Yes

Ever incarcerated

No

Yes

Length of opioid use history

15 Years of less

16-26 years

27-35 years

36 years or longer

OUD severity

Mild/moderate

Severe

Ever injected heroin

No

Yes

Cocaine use in past 30 days

No

Yes

Crack use in past 30 days

No

Yes

Marijuana use in past 30 days

No

Yes

Benzodiazepine use in past 30 days

No

Yes

Fentanyl detected in urine analysis

No

Yes

$146(58.4)$

219 (68.6)

59 (56.2)

$153(71.2)$

$139(61.5)$

$11(61.1)$

$117(61.6)$

$248(66.1)$

$289(65.5)$

$70(57.8)$

$264(66.7)$

$101(58.4)$

$74(66.7)$

$291(63.5)$

89 (58.6)

88 (65.2)

92 (62.8)

97 (71.3)

67 (72.0)

271 (62.2)

255 (70.8)

98 (50.3)

196 (68.8)

$169(59.5)$

265 (65.0)

$100(62.1)$

235 (66.2)

$130(60.8)$

254 (66.5)

$111(59.4)$

$184(68.9)$

$179(59.7)$

\section{Ref \\ $1.18(1.03,1.34)$}

Ref

$1.27(1.05,1.53)$

$1.09(0.90,1.33)$

$1.09(0.72,1.63)$

Ref

$1.07(0.94,1.23)$

Ref

$0.88(0.75,1.04)$

Ref

$0.88(0.76,1.01)$

Ref

$0.95(0.82,1.11)$

Ref

$1.11(0.93,1.34)$

$1.07(0.89,1.29)$

$1.22(1.03,1.44)$

Ref

$0.86(0.74,1.00)$

Ref

$0.71(0.61,0.83)$

Ref

$0.86(0.76,0.98)$

Ref

$0.96(0.83,1.10)$

Ref

$0.92(0.80,1.05)$

Ref

$0.89(0.78,1.02)$

Ref

$0.86(0.76,0.98)$
105 (41.3)

Ref

$80(24.9)$

$0.60(0.47,0.77)$

45 (42.9)

58 (26.7)

78 (34.1)

$3(15.8)$

Ref

$0.62(0.46,0.85)$

$0.79(0.60,1.06)$

$0.37(0.13,1.07)$

58 (30.2)

Ref

$1.07(0.83,1.39)$

132 (29.7)

Ref

$53(42.7)$

$1.44(1.12,1.84)$

$133(33.2)$

52 (29.9)

Ref

$0.90(0.69,1.18)$

$36(31.6)$

Ref

$149(32.3)$

$1.02(0.76,1.38)$

66 (42.9)

Ref

41 (30.2)

48 (32.6)

$0.70(0.51,0.96)$

$0.76(0.57,1.02)$

$0.51(0.35,0.74)$

14 (14.7)

Ref

$159(36.1)$

$2.45(1.49,4.04)$

107 (29.3)

Ref

75 (38.5)

$1.31(1.03,1.66)$

82 (28.6)

Ref

103 (35.8)

$1.25(0.98,1.59)$

121 (29.2)

Ref

64 (39.8)

$0.96(0.83,1.10)$

96 (26.9)

Ref

89 (40.8)

$1.52(1.20,1.92)$

105 (27.2)

Ref

$80(42.3)$

$1.56(1.23,1.96)$

89 (33.2)

Ref

$95(31.2)$
$0.94(0.74,1.19)$ 
Table 2 (continued)

\begin{tabular}{|c|c|c|c|c|}
\hline & $\begin{array}{l}N(\%) \text { with } \\
\text { unprotected use }\end{array}$ & $\begin{array}{l}\mathrm{PR}(95 \% \mathrm{Cl}) \text { for association } \\
\text { unprotected use }\end{array}$ & $\begin{array}{l}N(\%) \text { with OD } \\
\text { experience }\end{array}$ & $\begin{array}{l}\text { PR }(95 \% \mathrm{Cl}) \text { for } \\
\text { association with OD } \\
\text { experience }^{\mathrm{a}}\end{array}$ \\
\hline \multicolumn{5}{|c|}{ Oxycodone detected in urine analysis } \\
\hline No & $344(63.9)$ & Ref & $172(31.8)$ & Ref \\
\hline Yes & $19(65.5)$ & $1.02(0.78,1.34)$ & $12(37.5)$ & $1.18(0.74,1.88)$ \\
\hline \multicolumn{5}{|l|}{ Heroin detected in urine analysis } \\
\hline No & $49(62.0)$ & Ref & $32(40.5)$ & Ref \\
\hline Yes & $314(64.3)$ & $1.04(0.86,1.25)$ & $152(30.8)$ & $0.76(0.56,1.02)$ \\
\hline \multicolumn{5}{|l|}{ Stigma (median $\geq 13$ ) } \\
\hline No & $167(60.5)$ & Ref & $84(30.3)$ & Ref \\
\hline Yes & $189(68.5)$ & $1.13(1.00,1.28)$ & $96(34.3)$ & $1.13(0.89,1.44)$ \\
\hline \multicolumn{5}{|l|}{ PTSD } \\
\hline No & $330(65.0)$ & Ref & $161(31.4)$ & Ref \\
\hline Yes & $35(57.4)$ & & $24(38.7)$ & $1.23(0.88,1.73)$ \\
\hline \multirow[t]{2}{*}{ Omnibus mental health* } & & Ref & & Ref \\
\hline & & $0.95(0.86,1.04)$ & & $1.30(1.14,1.48)$ \\
\hline \multicolumn{5}{|l|}{ Depression } \\
\hline No & $172(65.9)$ & Ref & $66(24.9)$ & Ref \\
\hline Yes & $193(62.7)$ & $0.95(0.84,1.08)$ & $119(38.4)$ & $1.54(1.20,1.98)$ \\
\hline \multicolumn{5}{|l|}{ Anxiety } \\
\hline No & $210(67.5)$ & Ref & $89(28.2)$ & Ref \\
\hline Yes & $155(60.1)$ & $0.89(0.78,1.01)$ & $96(36.9)$ & $1.31(1.03,1.66)$ \\
\hline \multicolumn{5}{|l|}{ Suicidality } \\
\hline No & $349(64.4)$ & Ref & $170(31.1)$ & Ref \\
\hline Yes & $16(59.3)$ & $0.92(0.67,1.27)$ & $15(53.6)$ & $1.72(1.19,2.49)$ \\
\hline \multicolumn{5}{|l|}{ SEP visits in past 3 months } \\
\hline No & $251(73.4)$ & Ref & $95(27.4)$ & Ref \\
\hline Yes & $80(47.3)$ & $0.64(0.54,0.76)$ & $64(37.6)$ & $1.38(1.06,1.78)$ \\
\hline \multicolumn{5}{|l|}{ Currently opioid treatment } \\
\hline No current treatment & $161(64.1)$ & Ref & $99(39.1)$ & Ref \\
\hline Medication assisted treatment & $204(64.2)$ & $1.00(0.88,1.13)$ & $86(26.7)$ & $0.68(0.54,0.86)$ \\
\hline \multicolumn{5}{|l|}{ Ever trained to administer naloxone } \\
\hline No & $162(85.3)$ & Ref & $51(26.4)$ & Ref \\
\hline Yes & $200(53.3)$ & $0.62(0.56,0.70)$ & $132(34.9)$ & $1.32(1.00,1.74)$ \\
\hline \multicolumn{5}{|c|}{ Administered naloxone in past 3 months } \\
\hline No & $325(69.3)$ & Ref & $135(28.5)$ & Ref \\
\hline Yes & $40(40.0)$ & $0.58(0.45,0.74)$ & $50(49.5)$ & $1.74(1.36,2.22)$ \\
\hline
\end{tabular}

${ }^{\mathrm{a}}$ May not sum to 575 due to missing values

* Median (IQR); scale is standardized

(PR: 1.31; 95\% CI 1.03, 1.66). There is also evidence that overdose experience was more common among those who used other substances including cocaine (PR: 1.25 , 95\% CI 0.98, 1.59), marijuana (PR: 1.52 ; $95 \%$ CI $1.20,1.92$ ) and benzodiazepines (PR: $1.56,95 \%$ CI 1.23, 1.96). Overdose experience was strongly associated with suicidality (PR: 1.72, 95\% CI 1.19, 2.49) and also was associated with symptoms of depression (PR: 1.54:
95\% CI 1.20, 1.98) and anxiety (PR: $1.31,95 \%$ CI 1.03, 1.66). Those who were trained in and who had administered naloxone also were more likely to have more overdose experiences (trained: PR: 1.32, 95\% CI 1.00, 1.74); (administered: PR: 1.74, 95\% CI 1.36, 2.22).Those who tested positive for fentanyl did not have elevated overdose experiences compared with those who tested negative (PR: 0.94; 95\% CI 0.74, 1.19). 


\section{Associations: network and support factors and $0 \%$ protection (lacks naloxone and/or person to administer it) during opioid use (Table 3 )}

\section{Network size}

In both unadjusted analyses and in multivariable analyses adjusting for age, sex, race/ethnicity, current homelessness, and OUD severity, among injectors, having no person in one's injection network was associated with moderate increases in the prevalence of having no protection during opioid use events (APR: 1.61, 95\% CI 1.23, 2.11) (Table 4). In the sample overall, when including injecting and non-injecting participants, the APR for the association between small network size ( $<5$ persons) and never being protected during opioid use was 1.14 (95\% CI $0.98,1.31)$.

\section{Cohabitation romantic and non-romantic partners}

There was evidence that cohabitation with a romantic partner is protective against having $0 \%$ protected opioid use events (APR: 0.89, 95\% CI 0.76, 1.04). Living with a partner who also uses opioids was associated with a $23 \%$ reduction in the hazard of having no protection during opioid use (APR: 0.77, 95\% CI 0.62, 0.95). While there was a weak and non-significant relationship between living with anyone and protected events (APR: 0.99, 95\% CI $0.85,1.15)$, living with anyone who uses opioids was associated with having more than $25 \%$ more protected use events.

\section{Associations: network and support factors and overdose (Table 4) \\ Network size}

In both unadjusted and adjusted analyses, among injectors, there was little evidence of an association between network size and overdose risk.

\section{Cohabitation romantic and non-romantic partners}

Cohabitation with a romantic partner, with a partner who also uses opioids, or with anyone was not associated with overdose experiences.

\section{Discussion}

Overall, our results shed new light on social networks of people who use drugs, as participants in our study with larger social networks had greater coverage of naloxone and a person to administer it during opioid use events, what we have called "protected use," than participants with smaller networks. Having larger social networks of people who use opioids has the potential to result in a higher proportion of protected use events. By the same token, our finding that $45 \%$ of all participants reported that naloxone was never available during past-month opioid use events attests to the challenges we continue to face in trying to tackle this crisis and ensure that for every use of opioids, there is a naloxone kit and someone to administer it nearby.

Participants with larger social networks were considerably more likely to have protected use events, networks that have potential to provide more support to mitigate some of the hazards associated with solitary injection drug use and overdose response-a network member can administer naloxone in a timely way. Our finding that larger networks were not associated with overdose experiences in past 30 days, but were associated with ever being trained in naloxone use and having administered the medication in the past three months, supports other research showing the positive benefits of saturating communities with naloxone and ensuring the medication makes into the hands of people who use drugs [34-36]. The comparatively low prevalence of overdose experience among people who inject drugs who have no network members may be, among other factors, an indication that people who inject drugs alone are aware of having no potentially life-saving support and use with greater caution. Especially when using alone, these people who inject drugs may more readily use smaller amounts, check drug supplies for fentanyl using test strips distributed by harm reduction agencies, and/or do "test shots" to determine potency, all features of THN curricula. Future research is needed to better understand strategies deployed by solitary users to stay safe when they use alone, and if they are more cautious in solitary use situations.

Living with anyone who uses opioids, including a romantic partner, was associated with more naloxone protection but not more overdose experiences than among those who live alone or with a non-opioid user. This is yet another indication that naloxone distribution efforts need to also focus on getting naloxone into the extended networks of people who use drugs, users and non-users. But it also suggests that more targeted outreach to non-users who may live or interact with people who use drugs is needed. That one quarter (140 participants) of our sample lived alone, 33\% reported an overdose experience in the past 30 days, and $45 \%$ reported being never protected in the previous month of opioid use, suggests that the goal of being protected $100 \%$ of the time by naloxone and someone to administer it demonstrates the work ahead to connect people to protective networks and contexts for use (i.e., supervised spaces).

In the United States, the primary means of supervised drug consumption stems from one's network and the public, as interventions like safe consumption sites (SCSs) or Overdose Prevention Centers (OPCs) remain illegal at the federal level, placing the burden of overdose response one's social networks, often carried by 
Table 3 Associations between network and support factors and never protected with naloxone and a person to administer during opioid use events in past 30 days

\begin{tabular}{|c|c|c|c|}
\hline & $N(\%)$ with no protection & PR $(95 \% \mathrm{Cl})$ & $\operatorname{APR}(95 \% \mathrm{CI})^{\mathrm{a}}$ \\
\hline \multicolumn{4}{|l|}{ Network size (injecting) } \\
\hline More than 1 & $64(43.8)$ & Ref & Ref \\
\hline 0 in Network & $38(69.1)$ & $1.58(1.22,2.03)$ & $1.61(1.23,2.11)$ \\
\hline \multicolumn{4}{|l|}{ Network size (opioid-using) } \\
\hline More than 5 & $275(62.5)$ & Ref & Ref \\
\hline$<5$ in network & $90(69.8)$ & $1.37(0.90,2.09)$ & $1.14(0.98,1.31)$ \\
\hline \multicolumn{4}{|l|}{ Network size (opioid using, excluding injectors) } \\
\hline More than 5 & $201(71.3)$ & Ref & Ref \\
\hline$<5$ in Network & $62(72.10$ & $1.01(0.87,1.18)$ & $1.03(0.88,1.21)$ \\
\hline \multicolumn{4}{|c|}{ Non-using network gave place to stay in past 3 months } \\
\hline No & $248(65.1)$ & Ref & Ref \\
\hline Yes & $117(63.2)$ & $0.97(0.85,1.11)$ & $1.08(0.93,1.24)$ \\
\hline \multicolumn{4}{|l|}{ Non-using network gave money in past 3 months } \\
\hline No & $133(62.7)$ & Ref & Ref \\
\hline Yes & $229(64.9)$ & $1.03(0.91,1.18)$ & $1.07(0.93,1.22)$ \\
\hline \multicolumn{4}{|c|}{ Non-using network gave emotional support in past 3 months } \\
\hline No & $80(61.1)$ & Ref & Ref \\
\hline Yes & $281(65.2)$ & $1.06(0.92,1.24)$ & $1.17(0.99,1.38)$ \\
\hline \multicolumn{4}{|l|}{ Using network gave place to stay in past 3 months } \\
\hline No & $267(65.4)$ & Ref & Ref \\
\hline Yes & $98(61.2)$ & $0.94(0.81,1.08)$ & $1.03(0.88,1.20)$ \\
\hline \multicolumn{4}{|l|}{ Using network gave money in past 3 months } \\
\hline No & $211(68.5)$ & Ref & Ref \\
\hline Yes & $154(59.5)$ & $0.87(0.76,0.98)$ & $0.85(0.75,0.98)$ \\
\hline \multicolumn{4}{|c|}{ Using network gave emotional support in past 3 months } \\
\hline No & $144(72.0)$ & Ref & Ref \\
\hline Yes & $221(60.2)$ & $0.84(0.74,0.94)$ & $0.87(0.76,0.99)$ \\
\hline \multicolumn{4}{|l|}{ Support from networks } \\
\hline No support & $41(69.5)$ & Ref & Ref \\
\hline At least one form of support from using & $16(48.5)$ & $0.70(0.47,1.03)$ & $0.70(0.47,1.04)$ \\
\hline At least one form of support from non-using & $174(62.6)$ & $0.98(0.80,1.19)$ & $1.00(0.82,1.24)$ \\
\hline At least one from of support from both & $132(68.0)$ & $0.90(0.74,1.09)$ & $0.95(0.77,1.16)$ \\
\hline \multicolumn{4}{|l|}{ Currently lives with romantic partner } \\
\hline No & $277(65.8)$ & Ref & Ref \\
\hline Yes & $88(59.5)$ & $0.90(0.78,1.05)$ & $0.89(0.76,1.04)$ \\
\hline \multicolumn{4}{|l|}{ Currently lives with romantic partner who uses opioids } \\
\hline No & $312(67.0)$ & Ref & Ref \\
\hline Yes & $53(51.5)$ & $0.77(0.63,0.94)$ & $0.77(0.62,0.95)$ \\
\hline \multicolumn{4}{|l|}{ Currently lives with anyone } \\
\hline No & $92(66.7)$ & Ref & Ref \\
\hline Yes & $272(63.3)$ & $0.95(0.83,1.09)$ & $0.99(0.85,1.15)$ \\
\hline \multicolumn{4}{|l|}{ Currently lives with anyone who uses opioids } \\
\hline No & $267(68.1)$ & Ref & Ref \\
\hline Yes & $82(52.6)$ & $0.77(0.66,0.91)$ & $0.79(0.66,0.94)$ \\
\hline \multicolumn{4}{|l|}{ Current living situation } \\
\hline Lives alone & $92(66.7)$ & Ref & Ref \\
\hline Not with anyone who uses opioids & $175(68.9)$ & $1.03(0.89,1.19)$ & $1.06(0.91,1.24)$ \\
\hline Someone who uses opioids & $82(52.6)$ & $0.79(0.65,0.95)$ & $0.82(0.67,1.01)$ \\
\hline
\end{tabular}

${ }^{a}$ Adjusted for age, sex, race/ethnicity, current homelessness, and OUD severity 
Table 4 Associations between network and support factors and any OD experiences in past 30 days

\begin{tabular}{|c|c|c|c|}
\hline & $N(\%)$ with OD experience & PR $(95 \% \mathrm{Cl})$ & $\operatorname{APR}(95 \% \mathrm{Cl})^{\mathrm{a}}$ \\
\hline \multicolumn{4}{|l|}{ Network size (injecting) } \\
\hline More than 1 & $65(44.5)$ & Ref & Ref \\
\hline 0 in Network & $15(27.3)$ & $0.61(0.38,0.99)$ & $0.80(0.51,1.26)$ \\
\hline \multicolumn{4}{|l|}{ Network size (opioid using) } \\
\hline More than 5 & $143(32.3)$ & Ref & Ref \\
\hline$<5$ in Network & $42(32.1)$ & $0.99(0.75,1.32)$ & $1.04(0.79,1.36)$ \\
\hline \multicolumn{4}{|l|}{ Network size (opioid using, excluding injectors) } \\
\hline More than 5 & $79(27.7)$ & Ref & Ref \\
\hline$<5$ in Network & $26(29.2)$ & $1.05(0.72,1.53)$ & $1.12(0.78,1.60)$ \\
\hline \multicolumn{4}{|c|}{ Non-using network gave place to stay in past 3 months } \\
\hline No & $104(26.9)$ & Ref & Ref \\
\hline Yes & $80(43.0)$ & $1.60(1.26,2.02)$ & $1.42(1.10,1.83)$ \\
\hline \multicolumn{4}{|l|}{ Non-using network gave money in past 3 months } \\
\hline No & $60(27.9)$ & Ref & Ref \\
\hline Yes & $122(34.3)$ & $1.23(0.95,1.59)$ & $1.05(0.81,1.36)$ \\
\hline \multicolumn{4}{|c|}{ Non-using network gave emotional support in past 3 months } \\
\hline No & $29(21.8)$ & Ref & Ref \\
\hline Yes & $152(34.9)$ & $1.60(1.13,2.27)$ & $1.41(0.99,2.00)$ \\
\hline \multicolumn{4}{|l|}{ Using network gave place to stay in past 3 months } \\
\hline No & $111(26.9)$ & Ref & Ref \\
\hline Yes & $73(45.3)$ & $1.69(1.34,2.13)$ & $1.52(1.18,1.95)$ \\
\hline \multicolumn{4}{|l|}{ Using network gave money in past 3 months } \\
\hline No & $92(29.4)$ & Ref & Ref \\
\hline Yes & $92(35.4)$ & $1.20(0.95,1.53)$ & $1.22(0.96,1.55)$ \\
\hline \multicolumn{4}{|c|}{ Using network gave emotional support in past 3 months } \\
\hline No & $54(26.7)$ & Ref & Ref \\
\hline Yes & $129(34.8)$ & $1.30(1.00,1.70)$ & $1.27(0.96,1.67)$ \\
\hline \multicolumn{4}{|l|}{ Support from networks } \\
\hline No support & $8(13.3)$ & Ref & Ref \\
\hline At least one form of support from using & $13(39.4)$ & $2.95(1.36,6.40)$ & $2.79(1.31,5.93)$ \\
\hline At least one form of support from non-using & $57(28.9)$ & $2.17(1.10,4.29)$ & $1.89(0.97,3.67)$ \\
\hline At least one form of support from both & $104(37.1)$ & $2.78(1.43,5.41)$ & $2.30(1.20,4.39)$ \\
\hline \multicolumn{4}{|l|}{ Currently lives with romantic partner } \\
\hline No & $133(31.3)$ & Ref & Ref \\
\hline Yes & $52(34.7)$ & $1.11(0.85,1.45)$ & $1.11(0.95,1.46)$ \\
\hline \multicolumn{4}{|c|}{ Currently lives with romantic partner who uses opioids } \\
\hline No & $148(31.4)$ & Ref & Ref \\
\hline Yes & $37(35.9)$ & $1.14(0.86,1.53)$ & $1.00(0.72,1.39)$ \\
\hline \multicolumn{4}{|l|}{ Currently lives with anyone } \\
\hline No & $41(29.3)$ & Ref & Ref \\
\hline Yes & $144(33.2)$ & $1.13(0.85,1.51)$ & $1.02(0.75,1.39)$ \\
\hline \multicolumn{4}{|l|}{ Currently lives with anyone who uses opioids } \\
\hline No & $121(30.4)$ & Ref & Ref \\
\hline Yes & $58(37.2)$ & $1.22(0.95,1.57)$ & $1.09(0.83,1.44)$ \\
\hline \multicolumn{4}{|l|}{ Current living situation } \\
\hline Lives alone Lives alone & $41(29.3)$ & Ref & Ref \\
\hline Not with anyone who uses opioids & $80(31.0)$ & $1.06(0.77,1.45)$ & $0.98(0.71,1.36)$ \\
\hline Someone who uses opioids & $58(37.2)$ & $1.27(0.91,1.76)$ & $1.08(0.75,1.54)$ \\
\hline
\end{tabular}

${ }^{a}$ Adjusted for age, sex, race/ethnicity, current homelessness, and OUD severity 
people without the institutional frameworks around them, which can take an emotional toll [37]. One known underground OPC in the US has demonstrated individual and social benefits, showcasing the viability of this proven intervention in Canada and Europe within U.S. contexts [38-40]. Recently, two sanctioned OPCs began operating "above ground" in NYC communities that have experienced high levels of overdose mortality. In just the first two months of operation, the OPC reported 124 onsite overdose interventions, a strong testament to their life saving potential and the need for large scale implementation across U.S. communities so people are always able to find low threshold protection. The high rates of overdose fatalities in private residences [11] are a strong reminder that THN adaptation for solitary living, housing and shelter systems are sorely needed to assist people who use drugs, including supervised use spaces easily accessible in residences and group trainings that may have the secondary function of helping to connect people after explaining the value of having a buddy system [41]. An environment free of stigma and judgement can only benefit naloxone uptake. Based on our findings, it is clear that additional tools are needed to help people who use drugs prevent an overdose in the first place (e.g. safer drug supply programs [42]), expedite response to overdose events (e.g. SCSs [43]), and establish better mechanisms of protection for solitary users they may need in the event of an overdose (e.g. housing overdose prevention sites [44]; technology-adapted tools [45]). Decriminalization of drugs might also address fluctuations in opioid tolerance resulting from incarceration, and address the surreptitious nature of drug use shaped by criminalization, resulting in fewer solitary opioid consumption events. Additionally, innovative safety measures developed during the height of the COVID-19 pandemic may also prove useful for minimizing overdose fatalities among solitary users including telemedicine monitoring programs, technology to link friends or just fellow-users via the phone or social media, and home visitation wellness programs [46].

Our findings related to race suggests that additional research is critical to understanding why communities of people of color who use opioids appear less protected by naloxone than other groups [42]. Our finding that Black participants were considerably less likely to have protected use events may reflect an ongoing broader lack of access to the medication and understandable hesitancy to engage harm reduction resources more generally in some communities due to legacies of harm done onto communities of people of color by medical institutions $[47,48]$. Black people who use opioids may face logistical and financial barriers to treatment [49] and may have had negative experiences with healthcare. These past experiences and poor quality care may reinforce mistrust, which may also be a barrier to naloxone uptake [50-52]. Limited access to naloxone may also be grounded in the history of community resistance to syringe exchange programs in some urban Black neighborhoods [46] as well as ongoing stigmatization, stereotyping, and criminal justice responses to drug use in Black communities [53]. Taken together, these phenomena point to the critical need for tailored harm reduction curricula and outreach, developed by and for communities of people of color, in addition to outreach to underserved communities guided by community members. Peer-based programs may be of particular value [48].

For those persons who are 50 years or older, while they are less likely to have to have small networks, they do not have the same protection found in the full sample, where large networks come with greater protection (or less unprotectedness). Thus, for those who are 50 or older, despite being less likely to have smaller networks, they are also less likely to be protected, which moves against the overall correlation between network size and protection. With an aging population of opioid users and a high proportion of our sample reporting solitary living, additional research is needed to better understand why larger networks don't afford older people the same naloxone protection as found with other groups.

Our findings that participants with mental health symptoms of depression, anxiety, and suicidality were considerably more likely to have experienced an overdose in the past 30 days is consistent with recent research that shows experiencing anxiety, suicidal ideation, and depression can be a risk factor for overdose [54, 55]. Additional overdose prevention and naloxone distribution may prove fruitful alongside mental health screenings. Also, further research on co-morbidities and overdose risk is clearly needed as are innovative outreach models to reach persons at high risk for overdose with mental health concerns.

We note several limitations in the current study, the first being the study was conducted in NYC, a city with an expansive naloxone distribution infrastructure, and the experiences of people who use opioids may not be generalizable to other areas. Data are self-report and there is the possibility of under and over reporting. Measuring non-fatal overdose is difficult and may be biased by the social contexts of use, in that those who use with others are more likely to learn of non-fatal ODs-characterized by collapse, unexpected loss of consciousness, fingers or lips turning blue, etc.-than those that occur in isolated, solitary-use settings. Our data on network size is limited to the size of participants' opioid-using networks, we did not collect data on the full size of all of participants' non opioid-using 
networks. Finally, it is possible that there is an underreporting of overdose experiences as a person may not recognize that he/she had experienced an overdose.

\section{Conclusion}

Considering the importance of protected use amid the ongoing dual overdose and COVID-19 crises, it is vital that the high levels of unprotected use events reported in our study be disseminated and discussed among people who use drugs, particularly among people of color who use drugs. Such conversations might lead to ensuring people at risk of fatal overdose know where to get naloxone, consider who trusted peers are to use drugs with, are aware that overdose prevention sites are now available above ground in New York City, and consider what protected drug use means in their lives. Agencies providing services to people who use drugs or community-based participatory action researchers might be best positioned to host such dialogues.

In this era of fentanyl, overdoses occur more frequently and rapidly than those involving only heroin. With fentanyl detected in over half of our sample (59\%) and reports of more fentanyl in the illicit drug supply, it is critical that opioid use events involve a bystander or readily available responder and easily accessible naloxone to make sure the incidence of overdose does not continue to climb. As communities face overdose fatigue, naloxone saturation remains as important as ever and our collective efforts are needed to expand the reach of naloxone distribution and help foster a climate where people carry the medication without fear of police repercussion and judgement by others [56]. Aiming to increase the naloxone community safety net is contingent on social network participation. As such, interventions to prevent and respond to overdoses particularly when using drugs alone are critical in the fight to end the fatal overdose crisis.

\section{Acknowledgements \\ The authors thank all the people who participated in the study and shared their expertise.}

\section{Authors' contributions}

ASB and LE conceived of and designed the study and secured funding. JS conducted the analyses. ASB drafted the manuscript. MK wrote the first draft of the results. All authors, ASB, JS, JMB, MK, AR, LH, CM, LE provided critical feedback and contributed to the editing and writing of subsequent drafts. All authors read and approved the final manuscript.

\section{Funding}

This material is based upon work supported by the U.S. National Institute on Drug Abuse (NIDA; 1R01DA046653). NIDA had no further role in the study design; in the collection, analysis and interpretation of data; in the writing of the report; or in the decision to submit the paper for publication.

\section{Availability of data and materials}

The datasets used and/or analyzed during the current study are available from the corresponding author on reasonable request.

\section{Declarations}

Ethics approval and consent to participate

This study was approved by the New York University School of Medicine Institutional Review Board.

\section{Consent for publication}

N/A.

\section{Competing interests}

The authors declare that they have no competing interests.

\section{Author details}

'School of Global Public Health, New York University, New York, USA. ${ }^{2}$ Center for Drug Use and HIV/HCV Research, New York University, New York, USA.

${ }^{3}$ Center for Opioid Epidemiology and Policy, Grossman School of Medicine, New York University, New York, USA. ${ }^{4}$ Centre for Drug Policy and Evaluation, Unity Health Toronto, 209 Victoria St, Toronto, ON M5B 1T8, Canada. ${ }^{5}$ Dornsife School of Public Health, Drexel University, Philadelphia, PA, USA.

Received: 13 October 2021 Accepted: 17 February 2022

Published online: 04 March 2022

\section{References}

1. CDC, Provisional Drug Overdose Death Counts 2021.

2. Ahmad F, Rossen L, Sutton P. Provisisonal drug overdose death counts 2021, National Center for Health Statistics.

3. McDonald R, Campbell ND, Strang J. Twenty years of take-home naloxone for the prevention of overdose deaths from heroin and other opioids conception and maturation. Drug Alcohol Depend. 2017;178:176-87.

4. Strang J, et al. Heroin overdose: the case for take-home naloxone. BMJ. 1996;312(7044):1435-6.

5. Wheeler $\mathrm{E}$, et al. Opioid overdose prevention programs providing naloxone to laypersons-United States, 2014. MMWR Morb Mortal Wkly Rep. 2015;64(23):631-5.

6. Davis C, Carr D. State legal innovations to encourage naloxone dispensing. J Am Pharm Assoc. 2017:57(2):S180-4.

7. Rando J, et al. Intranasal naloxone administration by police first responders is associated with decreased opioid overdose deaths. Am J Emerg Med. 2015;33(9):1201-4.

8. Bessen $\mathrm{S}$, et al. Barriers to naloxone use and acceptance among opioid users, first responders, and emergency department providers in New Hampshire, USA. Int J Drug Policy. 2019;74:144-51.

9. Yang C, Favaro J, Meacham MC. NEXT harm reduction: an online, mailbased naloxone distribution and harm-reduction program. Am J Public Health. 2021:66:e1-5.

10. Davidson PJ, et al. Fatal heroin-related overdose in San Francisco, 19972000: a case for targeted intervention. J Urban Health. 2003;80(2):261-73.

11. Siegler A, et al. Unintentional opioid overdose deaths in New York City, 2005-2010: a place-based approach to reduce risk. Int J Drug Policy. 2014;25(3):569-74.

12. Tobin $\mathrm{K}$, et al. Awareness and access to naloxone necessary but not sufficient: examining gaps in the naloxone cascade. Int J Drug Policy. 2018;59:94-7.

13. Bennett AS, et al. Reasons people who use opioids do not accept or carry no-cost naloxone: qualitative interview study. JMIR Form Res. 2020;4(12):e22411.

14. Dayton L, et al. More than just availability: who has access and who administers take-home naloxone in Baltimore, MD. PLOS ONE. 2019;14(11):224-686.

15. Heavey SC, et al. 'I have it just in case'-Naloxone access and changes in opioid use behaviours. Int J Drug Policy. 2018;51:27-35.

16. Papamihali $\mathrm{K}$, et al. Convenience and comfort: reasons reported for using drugs alone among clients of harm reduction sites in British Columbia, Canada. Harm Reduct J. 2020;17(1):1-11.

17. Bowles J, et al. "Generally, you get 86 'ed because you're a liability": An application of Integrated Threat Theory to frequently witnessed overdoses and social distancing responses. Soc Sci Med. 2020;66:113-90. 
18. Latkin CA, et al. Stigma and drug use settings as correlates of selfreported, non-fatal overdose among people who use drugs in Baltimore, Maryland. Int J Drug Policy. 2019;68:86-92.

19. Bonevski $B$, et al. Reaching the hard-to-reach: a systematic review of strategies for improving health and medical research with socially disadvantaged groups. BMC Med Res Methodol. 2014;14(1):42

20. Pierce TG. Gen-X Junkie: ethnographic research with young white heroin users in Washington, DC. Substance Use Misuse. 1999;34(14):2095-114.

21. Latkin CA, Hua W, Tobin K. Social network correlates of self-reported nonfatal overdose. Drug Alcohol Depend. 2004;73(1):61-7.

22. Heckathorn DD. Respondent-driven sampling: a new approach to the study of hidden populations. Soc Probl. 1997;44(2):174-99.

23. Heckathorn DD, Cameron CJ. Network sampling: from snowball and multiplicity to respondent-driven sampling. Ann Rev Sociol. 2017;43:101-19.

24. Pouget ER, et al. Recent overdose experiences in a community sample of military veterans who use opioids. J Drug Issues. 2017;6:66.

25. Darke S, Ross J, Hall W. Prevalence and correlates of the injection of methadone syrup in Sydney, Australia. Drug Alcohol Depend. 1996:43(3):191-8.

26. Darke S, Mattick RP, Degenhardt L. The ratio of non-fatal to fatal heroin overdose. Addiction. 2003;98(8):1169-71.

27. Association, A.P., Diagnostic and statistical manual of mental disorders $\left(\right.$ DSM- $5^{\circledR}$ ). 2013: American Psychiatric Pub.

28. Ross MW, et al. Stigma consciousness concerns related to drug use and sexuality in a sample of street-based male sex workers. Int I Sex Health. 2007;19(2):57-67.

29. Lang AJ, et al. Abbreviated PTSD Checklist (PCL) as a guide to clinical response. Gen Hosp Psychiatry. 2012;34(4):332-8.

30. Narrow WE, et al. DSM-5 field trials in the United States and Canada, Part III: development and reliability testing of a cross-cutting symptom assessment for DSM-5. Am J Psychiatry. 2013;170(1):71-82.

31. StataCorp, L. Stata survival analysis reference manual. 2017.

32. Zou G. A modified poisson regression approach to prospective studies with binary data. Am J Epidemiol. 2004;159(7):702-6.

33. Buels $R$, et al. JBrowse: a dynamic web platform for genome visualization and analysis. Genome Biol. 2016;17(1):1-12.

34. Bennett AS, et al. From peers to lay bystanders: findings from a decade of naloxone distribution in Pittsburgh, PA. J Psychoactive Drugs. 2018;50(3):240-6.

35. Madah-Amiri D, Gjersing L, Clausen T. Naloxone distribution and possession following a large-scale naloxone programme. Addiction. 2020;6:66.

36. Bird SM, Parmar MK, Strang J. Take-home naloxone to prevent fatalities from opiate-overdose: protocol for Scotland's public health policy evaluation, and a new measure to assess impact. Drugs Educ Prev Policy. 2015;22(1):66-76.

37. Mamdani Z, et al. "Running myself ragged": stressors faced by peer workers in overdose response settings. Harm Reduct J. 2021;18(1):18.

38. Kral AH, et al. Evaluation of an unsanctioned safe consumption site in the United States. N Engl J Med. 2020;383(6):589-90.

39. Kral AH, Davidson PJ. Addressing the nation's opioid epidemic: lessons from an unsanctioned supervised injection site in the US. Am J Prev Med. 2017:53(6):919-22.

40. Maghsoudi N, Bowles J, Werb D. Expanding access to diacetylmorphine and hydromorphone for people who use opioids in Canada. Can J Public Health. 2020;111(4):606-9.

41. Bardwell G, et al. Addressing intersecting housing and overdose crises in Vancouver, Canada: opportunities and challenges from a tenant-led overdose response intervention in single room occupancy hotels. J Urban Health. 2019;96(1):12-20.

42. Ivsins A, et al. Tackling the overdose crisis: the role of safe supply. Int J Drug Policy. 2020;80:102-769.

43. Potier $C$, et al. Supervised injection services: what has been demonstrated? A systematic literature review. Drug Alcohol Depend. 2014;145:48-68

44. Bardwell $G$, et al. Housing and overdose: an opportunity for the scale-up of overdose prevention interventions? Harm Reduct J. 2017;14(1):1-4

45. Schwartz DG, et al. Layperson reversal of opioid overdose supported by smartphone alert: a prospective observational cohort study. EClinicalMedicine. 2020;25:100474.

46. Bennett A, Elliott L. Naloxone's role in the national opioid crisis-past struggles, current efforts, and future opportunities. Transl Res. 2021;234:43-57.
47. Owczarzak J, et al. "We know the streets:" race, place, and the politics of harm reduction. Health Place. 2020;64:106376.

48. Eversman MH. "We Want a Living Solution": views of harm reduction programs in Black US communities. J Ethnicity Substance Abuse. 2015;14(2):187-207.

49. Ong AR, Lee S, Bonar EE. Understanding disparities in access to naloxone among people who inject drugs in Southeast Michigan using respondent driven sampling. Drug Alcohol Depend. 2020;206:107-743.

50. Bowles JM, Lankenau SE. "I Gotta Go With Modern Technology, So I'm Gonna Give 'em the Narcan": the diffusion of innovations and an opioid overdose prevention program. Qual Health Res. 2019;29(3):345-56.

51. LaVeist TA, Nickerson KJ, Bowie JV. Attitudes about racism, medical mistrust, and satisfaction with care among African American and White cardiac patients. Med Care Res Rev. 2000;57(1_suppl):146-61.

52. Merrill JO, et al. Mutual mistrust in the medical care of drug users: the keys to the "narc" cabinet. J Gen Intern Med. 2002;17(5):327-33.

53. Hinton E, Head L, Reed C. An unjust burden: The disparate treatment of Black Americans in the Criminal Justice System. 2018, Vera Institute of Justice: New York.

54. Suffoletto B, Zeigler A. Risk and protective factors for repeated overdose after opioid overdose survival. Drug Alcohol Depend. 2020;209:107-890.

55. Cleland CM, et al. Between-and within-person associations between opioid overdose risk and depression, suicidal ideation, pain severity, and pain interference. Drug Alcohol Depend. 2020;206:107-734.

56. Winstanley EL. The bell tolls for thee \& thine: compassion fatigue \& the overdose epidemic. Int J Drug Policy. 2020;66:102796.

\section{Publisher's Note}

Springer Nature remains neutral with regard to jurisdictional claims in published maps and institutional affiliations.

Ready to submit your research? Choose BMC and benefit from

- fast, convenient online submission

- thorough peer review by experienced researchers in your field

- rapid publication on acceptance

- support for research data, including large and complex data types

- gold Open Access which fosters wider collaboration and increased citations

- maximum visibility for your research: over $100 \mathrm{M}$ website views per year

At BMC, research is always in progress.

Learn more biomedcentral.com/submissions 